\title{
Raman renaissance in defense and security
}

\section{Robert Stokes}

Advances in hardware and in chemistry and nanotechnology are continually increasing the applicability of Raman spectroscopy in the defense and security sector.

Conventional Raman spectroscopy is established as a versatile technique for both 'point-and-shoot' identification and forensic analysis of many illicit materials, including drugs and explosives. ${ }^{1}$ A number of manufacturers now provide robust, portable handheld platforms that can be deployed in a variety of situations. ${ }^{2-4}$ At the other end of the cost scale, larger lab-based spectrometer systems offer mapping or imaging capability in addition to spectral resolution and sensitivity. Recent reports in the media of terrorist incidents and insurgent activity indicate a growing trend toward the use of improvised explosive devices (IEDs) that are often manufactured using inconsistent methods and equipment. Raman spectroscopy provides a simple method of identifying these compounds, their composites, and precursors. Because of contamination, some of the materials are challenging to detect by conventional means. Two fundamental difficulties with Raman spectroscopy are the inherent weakness of the scattering process and the fluorescent background present in some samples.

Raman spectroscopy is typically initiated by exposing a sample to monochromatic radiation from a laser. A spectrometer can then be used to collect and analyze the scattering, most of which has the same frequency as the incident light. However, a small component, $\sim 10^{-5}$ of the incident beam, comprises photons with one vibrational unit difference in energy. Since there are a number of vibrations in most molecules, a Raman spectrum consists of a number of bands that can be used to identify a particular species positively and often nondestructively. The efficiency of scattering from individual modes is governed by Raman selection rules, and the overall intensity is inversely proportional to the wavelength of the excitation source. Conversely, unwanted fluorescence is often excited at shorter wavelengths, which means that a trade-off has to be established. System design is dictated by the economic
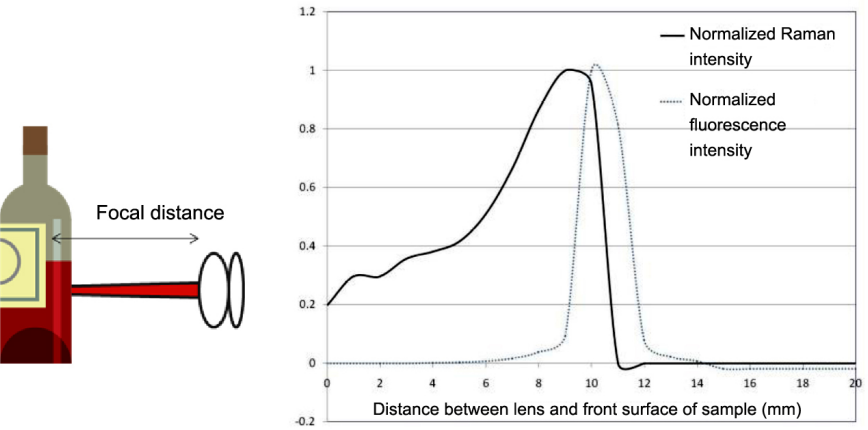

Figure 1. Focusing study using a typical confocal setup (focal distance of lens $=10.5 \mathrm{~mm}$ ). If the bottle is colored (e.g., most mineralwater bottles), a large fluorescent background is observed for excitation sources in the range of 500-750nm. (Reproduced with permission. ${ }^{5}$ )

costing performance of detectors and dispersion elements. Improvements in commercially available optical components enable rapid accumulation of signals at longer wavelengths of excitation. In addition, surface-enhanced Raman scattering (SERS) provides huge increases in effective scattering cross sections in the red and near-IR regions by tuning surface plasmon resonances to be coincident with incident and scattered photons.

Using a fast-scanning Raman system and recording over a limited spectral range, we have been able to identify potential components of improvised explosive devices and chemical weapons. ${ }^{5}$ Detailed study of the target molecules using both density functional theory (DFT) and high-resolution spectroscopy makes it possible to determine accurate fitting routines and parameters. In the cases of screening bottles and containers for target material, the choice of focusing optic is important. In a conventional setup, the optic must retain a sufficient numerical aperture to enable efficient collection with an adequate working distance to avoid interfering signals (both Raman and fluorescence) from the container wall. Figure 1 shows a typical focusing profile using hydrogen peroxide inside a fluorescent

Continued on next page 

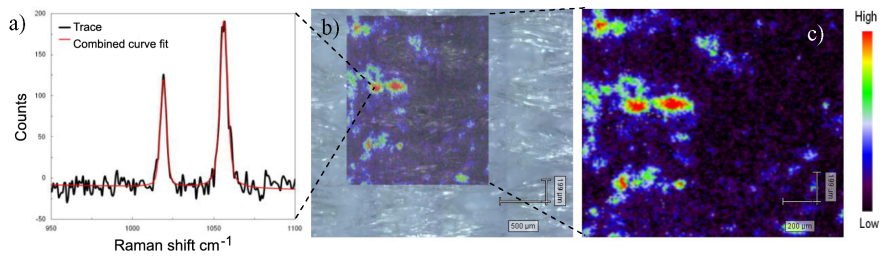

d)
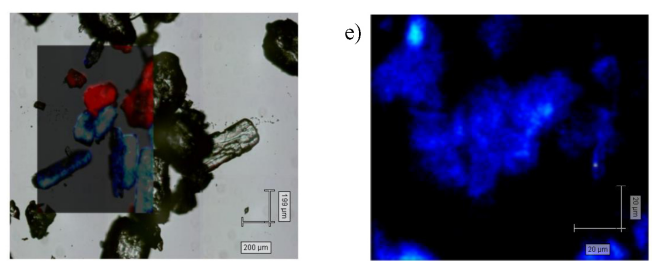

Figure 2. (a) Raw trace and total curve fit of the region corresponding to bands of urea nitrate, recorded under high speed (30Hz). (b) Montage brightfield image of white cord material doped with microcrystalline urea nitrate. The overlay is a Raman false-color image based on the intensity of the curve-fit data. (c) Magnified image of highlighted Raman map area. (d) Hyperspectral fast map recorded through a glass container wall showing urea (blue) and potassium nitrate (red) as separate components. (e) High-magnification Raman map of trace compounds of urea recorded though a glass barrier. (Reproduced with permission..$^{5}$ )

blue bottle as a target. Non-conventional optical arrangements, such as spatially offset Raman scattering, have also been shown to reduce the signals from the container wall. ${ }^{6}$

With a conventional optical setup and bespoke fitting algorithms, we have found that it is possible to record and identify hydrogen peroxide and ethanol at a scanning rate of 10 spectra per second. This is easily achieved by fitting the $v \mathrm{O}-\mathrm{O}$ stretching band of hydrogen peroxide $\left(\sim 872 \mathrm{~cm}^{-1}\right)$. The $\nu \mathrm{C}-\mathrm{C}-\mathrm{O}$ combined stretching band in ethanol $\left(869 \mathrm{~cm}^{-1}\right)$ is close enough to cause concern, as extremely low false-positive rates are paramount. Although a number of other relatively innocuous compounds have Raman bands that are in close proximity to the $v \mathrm{O}-\mathrm{O}$ band, accurate fitting routines ensure that misidentification is almost impossible under most illumination and background conditions. Using similar principles, routines can be generated for many other threat materials. The ability of Raman spectroscopy to identify chemical species sealed and in situ is important because many hazardous target materials rapidly decompose to harmless compounds on exposure to air and moisture.

Spectral identification routines can also be applied to rapid large-area mapping. Commercially available Raman linemapping systems such a Streamline ${ }^{\mathrm{TM}}$ (Renishaw, Wootenunder-edge, UK) can quickly generate large data sets from ex-

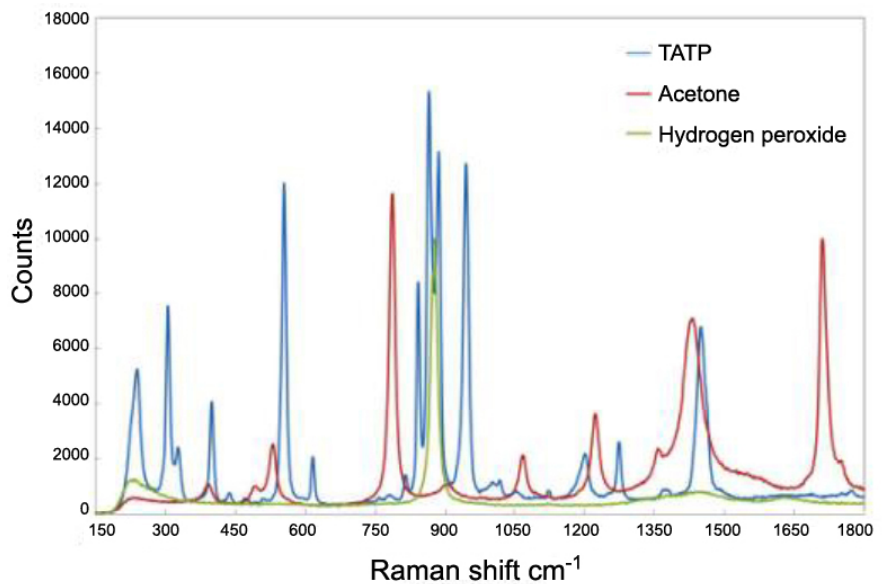

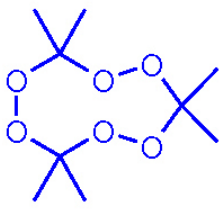

TATP<smiles>CC(C)=O</smiles>

Acetone

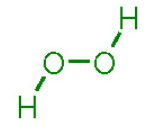

Hydrogen peroxide
Figure 3. The specificity of Raman scattering is shown by comparing the spectra of triacetonetriperoxide (TATP) with its precursors acetone and hydrogen peroxide under identical collection conditions.

tensive areas. ${ }^{5-7}$ These systems work by line-focusing the incident beam using a cylindrical lens and simultaneously collecting multiple spectra over the length of the area illuminated. Figure 2 shows an example of how we have used Raman line scanning coupled to spectral fitting routines to generate false-color images of IED materials rapidly and in situ. In this case, the improvised explosive urea nitrate (a white crystalline powder) is quickly detected from a background of white fabric. If the scanning system is adapted to incorporate optics suitable for bottle screening, identification of several materials can be made from inside a container (urea and potassium nitrate in this case).

This system has two benefits for the forensic analysis of explosives. First, the obvious increase in scanning speed allows larger areas to be covered within useful time periods. Second, and perhaps more important, the power density per unit area under the beam is lower than equivalent point scanning. This is important for explosives analysis, particularly some IED materials, because several are heat- and light-sensitive. A notable example is triacetonetriperoxide (TATP), which was used as a detonator by the 'shoe-bomber' 8 and in the London attacks of July $2005 .{ }^{9}$ The 


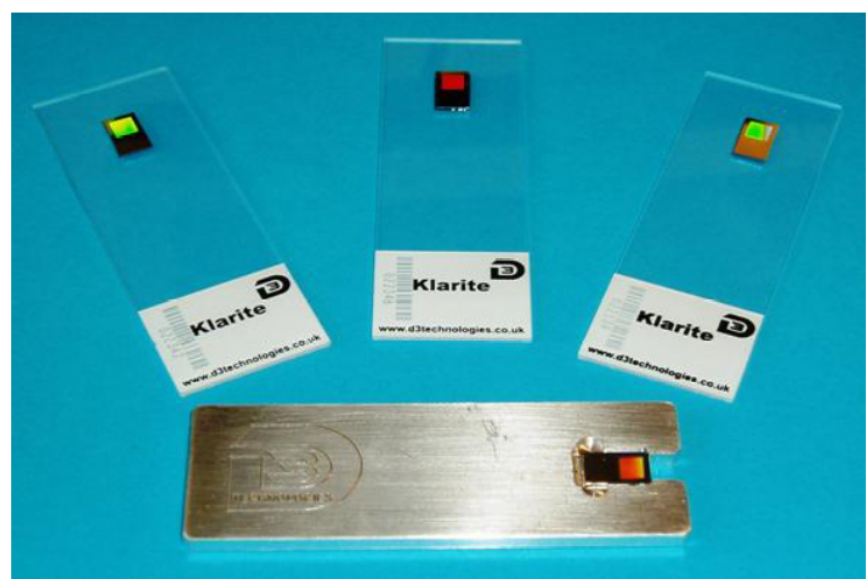

Figure 4. Klarite, the world's first bulk-manufactured SERS substrate, is available off-the-shelf and can detect trace levels of a range of illicit materials, such as drugs, explosives, and food contaminants.

highly specific Raman spectra of TATP (see Figure 3, bottom) is a good example of the information-rich nature of the technique.

In addition to rapid screening and forensic analysis, we have exploited Raman spectroscopy in micro- and nanoscience and technology for the highly sensitive identification of trace amounts of chemical and biological materials. ${ }^{10}$ For example, SERS uses areas of high electric field strength generated at the surface of certain nanoparticles and nanostructures. The result is a spectral response increased in sensitivity by $\sim 10^{6}$ times. A commercially available SERS surface, Klarite ${ }^{\mathrm{TM}}$ (D3 Technologies, Glasgow, UK), ${ }^{11}$ can identify many illicit materials, including most commonly used explosives. SERS is also able to detect anthrax spores ${ }^{12}$ and chemical weapons precursors. ${ }^{13}$ We recently found that modifying SERS surfaces either by thiol molecules or biological material can increase sensitivity and specificity, and reduce the standard deviation in signal intensity. In particular, chemical modification using gold-thiol chemistry and techniques such as dip-pen nanolithography ${ }^{14}$ can potentially allow quadratic increases in the number of potential targets that can be screened per unit area. ${ }^{15}$

Achieving usability is a major hurdle for many Raman-based techniques. Specifically, fast analysis and assessment of the threat must be very accurate (with a very low false-positive rate), and it must be packaged within a user-friendly, unambiguous interface. This may be achieved by improving fitting algorithms, spectral matching programs, and optical systems. Intelligent and accurate assessment of threats can also be made by 'fusing' Raman methods with technologies that have contrasting capability. SERS offers remarkably high sensitivity and selectivity, but often relies on the control of complex surface chemistry. The transition and simplification of the approach will enable further adoption within the defense and security sector.

\section{Author Information}

\section{Robert Stokes \\ Pure and Applied Chemistry \\ University of Strathclyde, WestCHEM \\ Glasgow, UK}

Rob Stokes is a member of the Centre for Molecular Nanometrology in Glasgow. His research focuses on applied spectroscopy and nanoscience for biomedical and defense applications. In the past two years he has written over 20 papers and three patents in these areas. He is also on the organizing committee for the SPIE conference titled Optics and Photonics for Counterterrorism and Crime Fighting V in Berlin in August 2009.

\section{References}

1. R. J. Stokes, K. Faulds, and W. E. Smith, Raman spectroscopy of illicit substances, Proc. SPIE 6741, p. 67410Q, 2007.

2. http://www.deltanu.com/ Delta nu. Accessed 4 February 2009.

3. http://www.ahurascientific.com/chemical-explosives-

id/products/firstdefender/index.php Ahura. Accessed 4 February 2009.

4. http://www.pacer.co.uk/jkcm/Products/Categories/Spectrometers/Raman

Pacer. Accessed 4 February 2009.

5. R. J. Stokes, W. E. Smith, B. E. Foulger, and C. Lewis, Rapid screening and identification of improvised explosive and hazardous precursor materials by Raman spectroscopy, Proc. SPIE 7119, pp. 711901-1, 2008.

6. C. Eliasson, N. A. Macleod, and P. Matousek, Non-invasive detection of concealed liquid explosives using Raman Spectroscopy, Anal. Chem. 79 (21), pp. 8185-8189, 2007

7. http://www.renishaw.com/en/6260.aspx Renishaw inVia Raman microscopes. Accessed 9 January 2009.

8. http://www.globalsecurity.org/military/systems/munitions/tatp.htm TATP factsheet. Accessed 9 January 2009.

9. http://en.wikipedia.org/wiki/21_July_2005_London_bombings

London terrorist incidents. Accessed 9 January 2009.

10. R. J. Stokes, A. Macaskill, J. A. Dougan, P. G. Hargreaves, H. M. Stanford, W. E. Smith, K. Faulds, and D. Graham, Highly sensitive detection of dye-labelled DNA using nanostructured gold surfaces, Chem. Commun. 27, pp. 2811-2813, 2007.

11. http://www.d3technologies.co.uk/sers_central/application_notes/index.html SERS application notes. See also http:/ / www.d3technologies.co.uk/applications / security.html Homeland security. Both accessed 9 January 2009.

12. X. Zhang, M. A. Young, O. Lyandres, and R. P. Van Duyne, Rapid detection of an anthrax biomarker by surface-enhanced Raman spectroscopy, J. Am. Chem. Soc. 127, pp. 4484-4489, 2005.

13. D. A. Stuart, K. B. Biggs, and R. P. Van Duyne, Surface-enhanced Raman spectroscopy of half-mustard agent, Analyst 131, pp. 568-572, 2006.

14. R. D. Piner, J. Zhu, F. Xu, S. Hong, and C. A. Mirkin, 'Dip-pen' nanolithography, Science 283, pp. 661-663, 1999.

15. R. J. Stokes, J. A. Dougan, and D. Graham, Dip-pen nanolithography and SERS as synergic techniques, Chem. Commun. 44, pp. 5734-5736, 2008. 